\title{
Detection and monitoring of subclinical ocular siderosis using multifocal electroretinogram
}

\author{
Pranita Sahay ${ }^{1} \cdot{\text { Devesh Kumawat } \mathbb{D}^{1} \cdot \text { Shikha Gupta } \mathbb{D}^{1} \cdot \text { Koushik Tripathy }^{2} \cdot \text { Rajpal Vohra }}^{1} \cdot$ Mahesh Chandra $^{1}$. \\ Pradeep Venkatesh ${ }^{1}$
}

Received: 10 April 2018 / Revised: 21 January 2019 / Accepted: 2 April 2019 / Published online: 24 April 2019

(c) The Royal College of Ophthalmologists 2019

\begin{abstract}
Background Although full-field electroretinogram (ffERG) is the gold standard test to detect physiological dysfunction in siderosis, it measures overall retinal function. This study aims to determine if multifocal electroretinogram (mfERG) can detect subclinical siderosis in eyes with an iron intraocular foreign body (IOFB).

Methods Twenty eyes of 20 patients with retained iron IOFB, clear ocular media and good visual acuity ( $\geq 20 / 120)$ were enroled in this prospective case-control study. The fellow eyes served as control. These were evaluated with ffERG and mfERG at baseline. Serial mfERG was done till six months after pars plana vitrectomy with IOFB removal. Primary outcomes measures were amplitude and peak time of P1 and N1 wave of mfERG.

Results The median age was 25 years (range 18-55). Most patients $(n=14 / 20)$ presented within a month of trauma. Baseline ffERG showed no difference in either ' $a$ ' or ' $b$ ' wave amplitude or peak time between cases and controls. However, on mfERG, there was a significant decrease in P1 and $\mathrm{N} 1$ wave amplitude and delay in P1 wave peak time in $<2^{\circ}$ retinal ring in cases as compared to controls ( $p=0.001,0.001$ and 0.02 respectively) despite variability in results. At 6 months, P1 amplitude showed significant improvement from baseline in cases $(p=0.010)$. However, P1 peak time did not show significant recovery $(p=0.65)$.

Conclusions mfERG may reveal subclinical electrophysiological retinal dysfunction in eyes with iron IOFB in cases with normal ffERG. P1 peak time may serve as an electrophysiological marker for past retinal damage.
\end{abstract}

\section{Introduction}

Siderosis bulbi is progressive deposition of iron due to retained iron intraocular foreign body (IOFB), leading to degeneration of the retina, development of cataract and secondary glaucoma in the late stages [1]. Siderotic changes of the retina become evident after a period of few months to few years [2].

Supplementary information The online version of this article (https:// doi.org/10.1038/s41433-019-0442-y) contains supplementary material, which is available to authorized users.

Pradeep Venkatesh

venkyprao@yahoo.com

1 Dr. Rajendra Prasad Centre for Ophthalmic Sciences, All India Institute of Medical Sciences, New Delhi, India

2 ASG Eye Hospital, Kolkata, West Bengal, India
Full-field electroretinogram (ERG) is the gold standard investigative modality to assess the retinal dysfunction in patients with IOFB. The development of changes in ERG has important prognostic implications and is an indication for surgical removal of the IOFB [3]. On ERG, initially a hyper-normal ' $a$ ' wave is seen followed by a steady decrease in the amplitudes of ' $a$ ' and ' $b$ ' waves. The origin of ' $a$ ' wave is predominantly photoreceptors, and 'b' wave is predominantly depolarising bipolar cells. The abovedescribed changes suggest retinal dysfunction in response to the ionic changes in outer retina [4].

While full-field ERG measures the mass potential (summed electrophysiological activity) of the retina [5], multifocal electroretinogram (mfERG) is a novel electrophysiological test developed to give a topographic measure of the electrophysiological activity at the macula [6]. mfERG has been found to be affected early in the course of various acquired retinal disorders like age related macular degeneration [7], diabetic retinopathy [8], idiopathic macular hole [9], drug toxicity-chloroquine/ 
hydroxychloroquine, ethambutol, sildenafil, vigabatrin [10], and hereditary retinal disorders affecting predominantly the macula like Stargardt's disease and Retinitis pigmentosa $[11,12]$.

Though ERG changes in eyes with retained iron IOFB are already documented in the literature [2-4], there is paucity regarding mfERG changes in eyes with iron IOFB. We had previously reported that the peak time of P1 wave of mfERG might be an early predictor of ferrous toxicity much before the onset of changes on ERG [13]. We conducted this study to further illustrate the changes in P1 and N1 waves of mfERG in eyes with iron IOFB at baseline and after surgical removal of the foreign body.

\section{Material and methods}

A prospective interventional comparative study was undertaken after obtaining approval from the Institute's Ethics Committee. The study adhered to the tenets of the Declaration of Helsinki. In this pilot study, twenty eyes of twenty consecutive patients presenting to the outpatient department with an antecedent history of trauma and evidence of retained iron IOFB on non-contrast computed tomography (CT) orbit were enroled. Fellow eye of the same patients served as control. Written informed consent was obtained from all patients before enrolment into the study.

The inclusion criteria for enrolment included retained iron IOFB, clear ocular media, ability to fixate, corrected distance visual acuity $($ CDVA) $\geq 20 / 20$, normal intraocular pressure $(\leq 21 \mathrm{mmHg})$ and patients willing to participate and available for all subsequent follow-ups. The exclusion criteria included corneal opacity, concurrent lenticular injury or traumatic cataract, vitreous haemorrhage, endophthalmitis, retinal detachment, macular scarring, and any pre-existing retinal disorder before the trauma in either eye or patients not willing to participate in the study.

The preoperative clinical assessment included CDVA on Snellen chart, slit lamp examination, intraocular pressure (NT-530P Non-contact tonometer, Nidek Technologies), fundus and vitreous evaluation (slit lamp biomicroscopy with + 90D noncontact lens) and dilated peripheral retinal examination (Indirect Ophthalmoscopy with $+20 \mathrm{D}$ noncontact lens). The investigations conducted included central macular thickness (Cirrus-HD OCT 4000; SD-OCT by Carl Zeiss Meditec AG, Jena, Germany), photopic and scotopic full-field electroretinogram (Vision monitor Mon 2012H Metrovision, France), multifocal electroretinogram (Vision monitor, Monpack 3, Metrovision, France) and non-contrast CT scan of the orbit. The site of injury to globe was noted and classified as per ocular trauma classification (OTC) group for open globe injury [14].

\section{ERG: Procedure}

Scotopic and photopic 3.0 ERG was performed separately on both eyes on Vision monitor Mon 2012H Metrovision. The recording was done using skin electrodes (silver/silver chloride cupula electrodes, Technomed, Europe). A positive electrode was placed over the lower eyelid and a negative electrode at the outer canthus. A ground electrode was placed at the ipsilateral earlobe. The skin was cleaned, and conductive paste (integral to the electrode) was applied to ensure good electrical connections. Full-field (Ganzfeld) stimulation was used to provide uniform luminance over the entire visual field in the dark-adapted and light adapted phase as per the guidelines given by International Society for Clinical Electrophysiology of Vision (ISCEV) [15]. Both ' $a$ ' and ' $b$ ' wave amplitude and peak time (also called implicit time) were recorded for scotopic and photopic ERG.

\section{MfERG: Procedure}

The readings were recorded from each eye separately on Vision monitor, Monpack 3, Metrovision as per the guidelines issued by ISCEV [16]. 61 scaled hexagonal stimulus patterns with central fixation point was used for stimulation. The recording was done using contact lens electrodes (ERG jet ${ }^{\circledR}$, Fabrinal SA) after anaesthetising the cornea with proparacaine $0.5 \%$ eye drop. The standard parameters recorded were $\mathrm{P} 1$ and $\mathrm{N} 1$ wave amplitude and their peak time. Both the waves were evaluated in five rings in the macular region with ring 1 representing $<2^{\circ}$ field, ring 2 representing $2-5^{\circ}$ field, ring 3 representing $5-10^{\circ}$ field, ring 4 representing $10-15^{\circ}$ field and ring 5 representing $>15^{\circ}$ field.

\section{Surgery}

All affected eyes underwent 25 gauge pars plana vitrectomy with IOFB removal through sclerotomy incision with the help of either intraocular forceps or magnet. Iso-expansile gas (18\% sulphur hexafluoride or $14 \%$ perfluoropropane) was used for endotamponade if there was no retinal tear/ detachment and 1000 centistokes silicone oil (Aurosil, Aurolab, India) was inserted in the event of any retinal tear/ detachment. Silicone oil was removed at 3 months after surgery in eyes in which it was used. The lens was preserved in all cases.

\section{Follow up schedule}

Follow-up of patients was done at day one, 1 week, 1 month, 3 months and 6 months after the surgery. The outcome measures at follow-up included 
CDVA, IOP, posterior segment evaluation (slit lamp biomicroscopy and indirect ophthalmoscopy) and recording mfERG for $\mathrm{P} 1$ and $\mathrm{N} 1$ wave amplitude and peak time.

\section{Statistical analysis}

Analysis of the data was done using IBM SPSS 20 software. CDVA was converted to logarithm of minimal angle of resolution (Log MAR). Group averages of P1 and N1 wave amplitude and peak time were analysed in each retinal ring. Groups with non-parametric data were subjected to 'Mann Whitney test' and to assess the changes in these groups over a period of time 'Friedman test' was used. Groups with parametric data were subjected to 'Two-sample t-test' and to see the changes in these groups over a period of time 'repeated measure analysis' was done. A $p$-value $\leq$ 0.05 was considered statistically significant.

\section{Results}

The median age of patients was 25 years (range-18 to 55). Majority of patients were in the age group of 20-40 years $(n=15 / 20)$. All patients were male. Hammer chisel injury was the mode of injury in all patients. Fourteen patients presented within 30 days of trauma while only five presented between 1 month to 6 months and one presented after two years (median time- 20 days, range -1 day to 2 years).

According to OTC, $95 \%$ of the injuries belonged to zone 1 (involving only cornea and limbus) and zone 2 (involving sclera up to $5 \mathrm{~mm}$ from limbus). The mean dimension of the removed foreign body was $4.1 \pm 0.8 \mathrm{~mm}$ with the range being 2.5 to $6 \mathrm{~mm}$. The foreign body was lying free in the vitreous cavity in seven eyes, encapsulated over the retina in 12 eyes and impinged in the ciliary body in one eye. None of the affected eyes had evidence of siderosis on slit lamp examination. The median time for surgery following patient presentation was 1 week. In $80 \%$ of the affected eyes $(n=16 /$ 20 ), iso-expansile gas was used for endo-tamponade while in the rest $20 \%$ eyes $(n=4 / 20)$, silicone oil was used. In three out of the four eyes in which silicone oil was used, the IOFB was found to be incarcerated in the retina while in one eye there was localised subretinal fluid around the FB.

\section{CDVA}

The baseline median Log MAR CDVA in affected eyes was 0.18 units (range 0.0-1.0). The Log MAR CDVA decreased significantly on post-operative follow up at 1 week with the median being 0.48 units (range 0.0-1.8) ( $p=0.02$, Wilcoxon signed rank test). However, CDVA returned to baseline from 1 month onwards.

\section{Central macular thickness}

The mean central macular thickness in affected and fellow eyes at baseline was $235.80 \pm 12.75 \mu \mathrm{m}$ and $231.05 \pm 9.40$ $\mu \mathrm{m}$ respectively with no statistically significant difference $(p=0.18$, two sample t-test).

\section{Electroretinogram}

At baseline, the mean amplitude and peak time of both ' $a$ ' and ' $b$ ' wave of both the scotopic and photopic full-field ERG were not significantly different in the affected and fellow eyes (Table 1).

\section{Multifocal electroretinogram}

\section{P1 wave}

In $<2^{\circ}$ ring, the baseline amplitude and peak time in affected eyes were 595.50 $\pm 282.70 \mathrm{nV}$ (cv 0.47) and 44.59 $\pm 7.40 \mathrm{msec}$ (cv 0.16 ) respectively and that in fellow eyes were $1167.30 \pm 464.70 \mathrm{nV}$ (cv 0.39 ) and $41.33 \pm 2.35 \mathrm{msec}$ (cv 0.06) respectively. The variance between the group was significantly different. The decrease in amplitude and delay in peak time in affected eyes were statistically different from the fellow eye at baseline $(p=0.001$ and 0.02 respectively). After removal of IOFB, at 6 months postoperatively, the amplitude in affected eyes improved significantly from baseline $(p=0.010)$ but remained significantly lower than that of fellow eyes $(p=0.006)$ (Table 2). The maximum improvement was noticed in the inner ring $\left(<2^{\circ}\right)$ in which the $\mathrm{P} 1$ wave mean amplitude increased by $30.4 \%$ while in the outer ring $\left(>15^{\circ}\right)$ the P1 wave mean amplitude increased by only $19.4 \%$. The peak time in affected eyes improved insignificantly on follow-up $(p=0.65)$ (Table 3).

When comparing the eyes of patients who presented late (>30 days from trauma, $n=14 / 20$ ) from those who presented early ( $<30$ days from trauma, $n=6 / 20$ ), the mean amplitude was less in late presenters $(429.60 \pm 95.88 \mathrm{nV})$ compared to those presenting early $(650.80 \pm 304.51 \mathrm{nV})$, as shown in Fig. 1a. Also, the late-presenting cases had a much prolonged mean peak time $(51.40 \pm 10.40 \mathrm{msec})$ than those who presented early $(42.32 \pm 4.75 \mathrm{msec})$, as shown in Fig. 1b. However, a statistical comparison could not be performed between these two groups due to an inadequate sample size of late presenters.

On analyzing the groups with different endo-tamponade used while surgery, the baseline mean amplitude and peak time in eyes where gas was used $(n=16 / 20)$, were 627.40 $\pm 305.00 \mathrm{nV}$ and $42.90 \pm 5.10 \mathrm{msec}$ respectively, while in eyes where silicone oil was used $(n=4 / 20)$, these were $468.00 \pm 114.60 \mathrm{nV}$ and $51.30 \pm 12.00 \mathrm{msec}$ respectively 


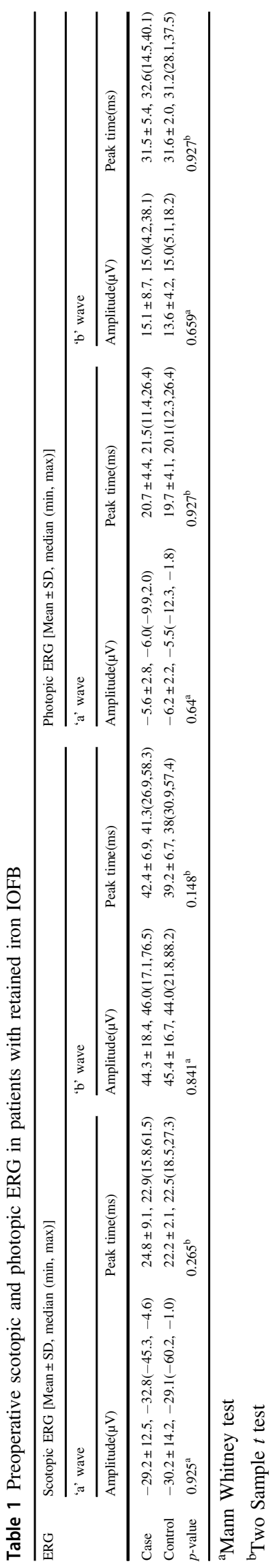

(Supplementary Fig 1 and 2). These could not be statistically compared between the two groups due to an inadequate sample size of oil group. Postoperatively the amplitude and peak time in oil group remained affected compared to the gas group. Following silicone oil removal at 3 months, there was an increase in amplitude and decrease in peak time in the oil group; though statistically insignificant ( $p=0.10$, Wilcoxon signed rank test and 0.50 , Two-sample t-test respectively).

Similar changes were seen in P1 wave amplitude and peak time in $2-5^{\circ}, 5-10^{\circ}, 10-15^{\circ}$ and $>15^{\circ}$ ring at baseline (Tables 2 and 3 respectively). The mean amplitude in affected eyes in $<2^{\circ}$ ring was $48.9 \%$ less compared to the fellow eyes $(p=0.001)$ while in the $>15^{\circ}$ ring the mean amplitude in the affected eyes was reduced only by $24 \%$ compared to that in fellow eyes $(p=0.02)$. The mean peak time in $<2^{\circ}$ ring in affected eyes was prolonged by $7.8 \%$ compared to that in fellow eyes $(p=0.001)$ while in $>15^{\circ}$ ring the mean peak time in affected eyes was prolonged by $13.4 \%$ compared to that in fellow eyes $(p=0.002)$. Maximum delay in the mean peak time was noted in the paracentral $2-5^{\circ}$ ring $(18.8 \%)$. Variability in data was noted in the affected group. A box plot representation of both $\mathrm{p} 1$ wave amplitude and peak time at presentation is presented in Fig. 2a, b. Despite the variability in results in the affected group, the values were significantly different from the control group. A scatter plot representation of the $\mathrm{p} 1$ wave amplitude and peak time in the $<2^{\circ}$ ring is depicted in Fig. 2c, d.

On follow-up, variable results were seen in different rings with outer rings showing no significant difference in the amplitude between the affected and fellow eyes at 6 months (Table 2). Also, the peak time in other rings did not change significantly on follow-up (Table 3 ).

\section{N1 Wave}

In $<2^{\circ}$ ring, the baseline amplitude and peak time in affected eyes were $-327.60 \pm 185.10 \mathrm{nV}$ and $23.70 \pm$ $6.10 \mathrm{msec}$ respectively and that in fellow eyes were $-652.00 \pm 298.10 \mathrm{nV}$ and $21.80 \pm 2.40 \mathrm{msec}$ respectively (supplementary Tables 1 and 2). The amplitude in affected eyes at baseline differed significantly from the fellow eyes $(p=0.001)$. However, the peak time did not show any statistical difference between the affected and fellow eyes $(p=0.24)$. At 6 months postoperatively, the amplitude improved from baseline $(p=0.94)$ but remained worse than that of fellow eyes $(p=0.001)$. Also, the peak time of affected eyes did not change on follow up $(p=0.57)$

Similar changes were seen in N1 wave amplitude in $2-5^{\circ}, 5-10^{\circ}, 10-15^{\circ}$ and $>15^{\circ}$ ring at baseline. In $<2^{\circ}$ ring the mean amplitude in affected eyes was decreased by $49.8 \%$ compared to fellow eyes $(p=0.001)$ while in $>15^{\circ}$ 


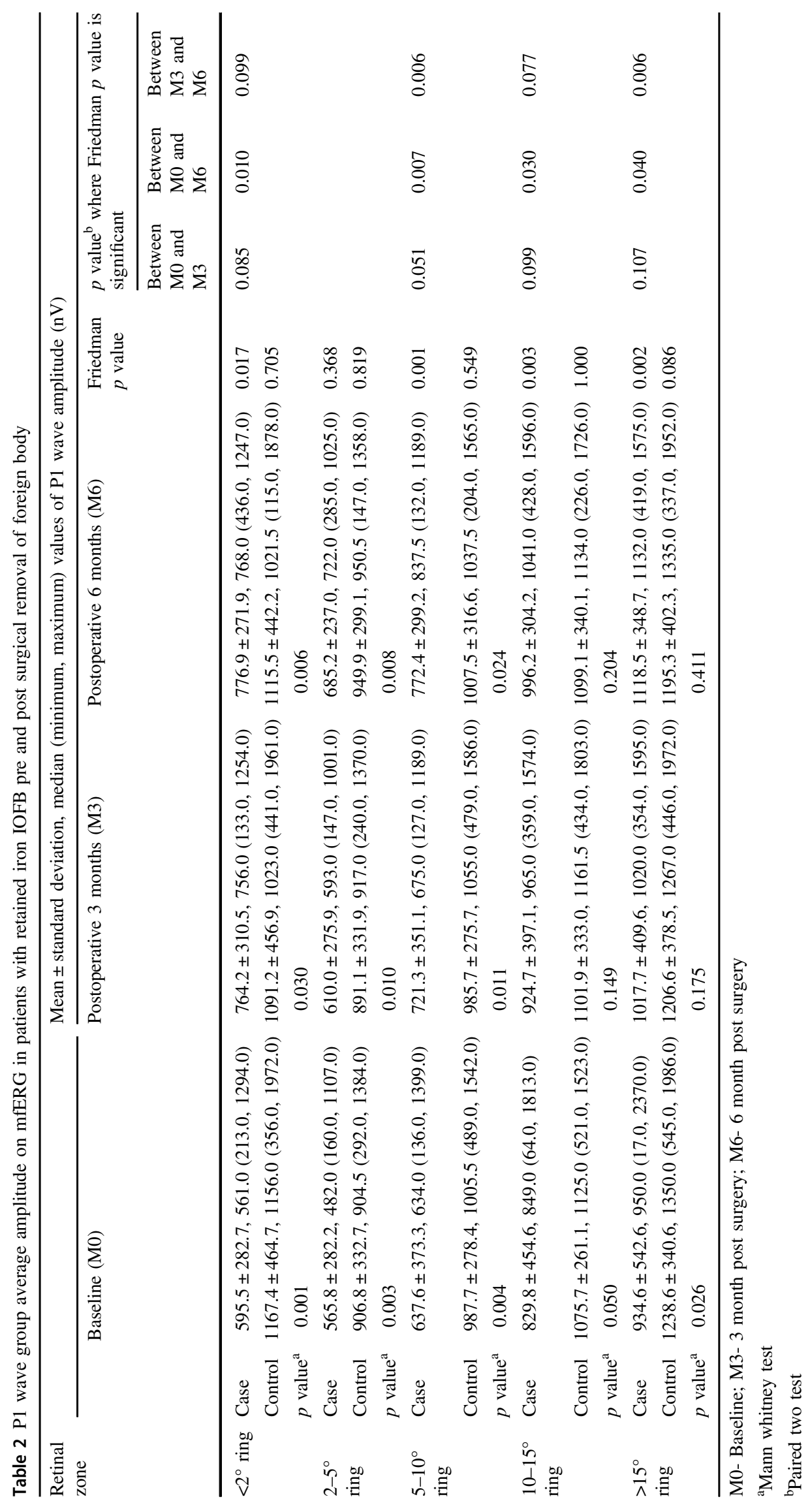


Table 3 P1 wave group average peak time on mfERG in patients with retained iron IOFB pre and post-surgical removal of foreign body

\begin{tabular}{|c|c|c|c|c|c|}
\hline \multirow{2}{*}{$\begin{array}{l}\text { Retinal } \\
\text { zone }\end{array}$} & & \multicolumn{4}{|c|}{ Mean \pm standard deviation values of P1 wave peak time (msec) } \\
\hline & & Baseline (M0) & $\begin{array}{l}\text { Postoperative } \\
3 \text { months (M3) }\end{array}$ & $\begin{array}{l}\text { Postoperative } \\
6 \text { months (M6) }\end{array}$ & $\begin{array}{l}p \text { value (Repeated } \\
\text { measure analysis) }\end{array}$ \\
\hline \multirow[t]{3}{*}{$<2^{\circ}$ ring } & Case & $44.59 \pm 7.40$ & $45.02 \pm 7.00$ & $44.08 \pm 6.82$ & 0.650 \\
\hline & Control & $41.33 \pm 2.30$ & $40.8 \pm 2.42$ & $42.13 \pm 4.81$ & 0.613 \\
\hline & $p$ value $^{\mathrm{a}}$ & 0.029 & 0.021 & 0.306 & \\
\hline \multirow[t]{3}{*}{$2-5^{\circ}$ ring } & Case & $48.04 \pm 10.11$ & $45.90 \pm 8.09$ & $44.04 \pm 6.18$ & 0.564 \\
\hline & Control & $40.42 \pm 1.72$ & $41.11 \pm 2.79$ & $41.36 \pm 2.83$ & 0.839 \\
\hline & $p$ value $^{\mathrm{a}}$ & 0.004 & 0.020 & 0.087 & \\
\hline \multirow[t]{3}{*}{$5-10^{\circ}$ ring } & Case & $44.47 \pm 6.05$ & $45.03 \pm 6.50$ & $43.80 \pm 3.97$ & 0.965 \\
\hline & Control & $40.63 \pm 1.35$ & $40.80 \pm 2.02$ & $40.73 \pm 2.23$ & 0.892 \\
\hline & $p$ value $^{\mathrm{a}}$ & 0.005 & 0.009 & 0.005 & \\
\hline \multirow{3}{*}{$\begin{array}{l}10-15^{\circ} \\
\text { ring }\end{array}$} & Case & $45.17 \pm 6.43$ & $44.80 \pm 7.69$ & $44.33 \pm 7.82$ & 0.512 \\
\hline & Control & $40.21 \pm 1.50$ & $40.80 \pm 2.12$ & $40.43 \pm 2.08$ & 0.933 \\
\hline & $p$ value $^{\mathrm{a}}$ & 0.004 & 0.030 & 0.038 & \\
\hline \multirow[t]{3}{*}{$>15^{\circ}$ ring } & Case & $45.7 \pm 6.50$ & $43.68 \pm 2.50$ & $43.6 \pm 3.70$ & 0.517 \\
\hline & Control & $40.3 \pm 2.10$ & $40.90 \pm 2.6$ & $40.60 \pm 2.30$ & 0.979 \\
\hline & $p$ value $^{\mathrm{a}}$ & 0.002 & 0.003 & 0.004 & \\
\hline
\end{tabular}

M0- Baseline; M3- 3 month post surgery; M6- 6 month post surgery

${ }^{\text {a }}$ Two sample $t$ test.

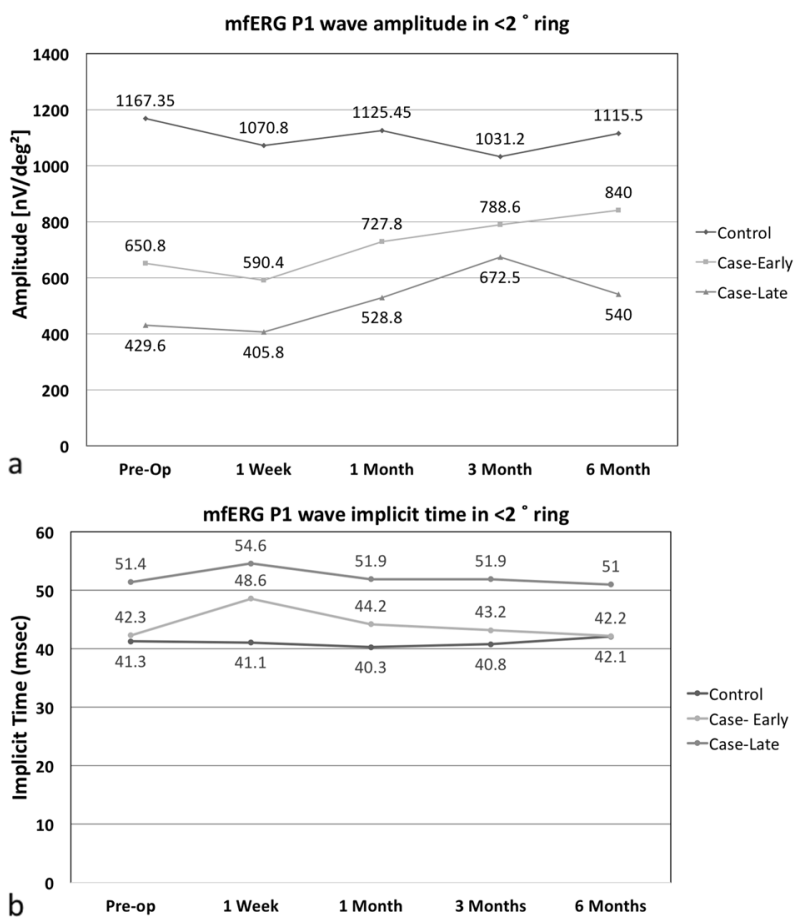

Fig. 1 P1 wave amplitude (a) and peak time (b) in $<2^{\circ}$ ring on multifocal electroretinogram (mfERG) in early and late cases pre and post-surgical removal of retained iron IOFB

ring the mean amplitude in affected eyes was reduced by $32.9 \%$ compared to fellow eyes $(p=0.001)$. The N1 peak time was significantly prolonged in affected eyes as compared to fellow eyes at baseline in these rings unlike in $<2^{\circ}$ ring. In $<2^{\circ}$ ring the mean peak time in affected eyes was prolonged by $8.5 \%$ compared to fellow eyes $(p=0.24)$. The paracentral ring $\left(2-5^{\circ}\right)$ showed the most prolonged peak time with respect to the fellow eyes. On follow-up, no significant change could be appreciated in amplitude or peak time in the affected eyes compared to baseline.

\section{Discussion}

Full-field ERG is the gold standard test to assess the retinal damage by an iron IOFB as it can pick up functional abnormalities of the retina before any pathological changes are visible in fundus or are picked up by fluorescein angiography [17].

In our study, both the scotopic and photopic ERG were essentially normal in affected eyes in terms of amplitude and peak time. Most patients had presented early, within a month after trauma and had normal fundus thereby indicating that the retinal damage might not have been significant enough to be picked by an ERG. Even the case that presented after two years of trauma did not have any siderotic changes in clinical evaluation. The absence of clinical changes may be due to encapsulated gliosis of the foreign body, which may have prevented siderosis from setting in, as has been described previously in the literature [18]. Full field ERG measures the overall retinal function and often small abnormal areas at macula may remain undetected [5]. mfERG selectively tests macular 


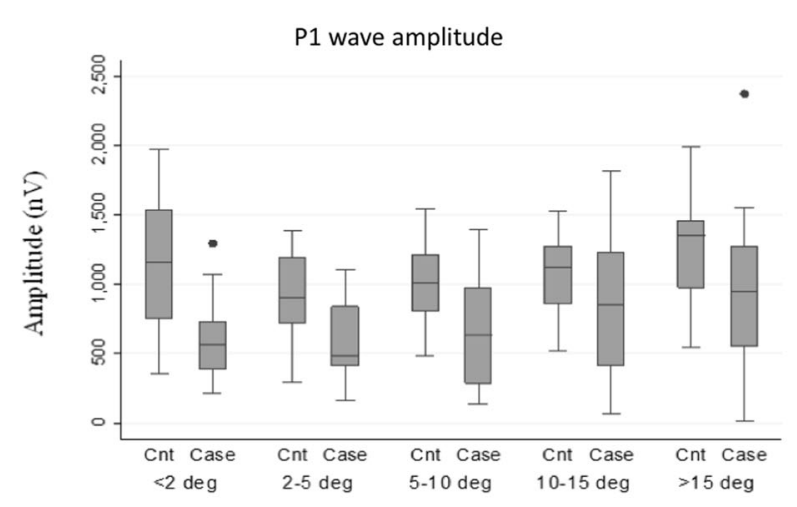

a

Retinal rings of $\mathrm{mfERG}$

P1 wave amplitude in $<2^{\circ}$ retinal ring

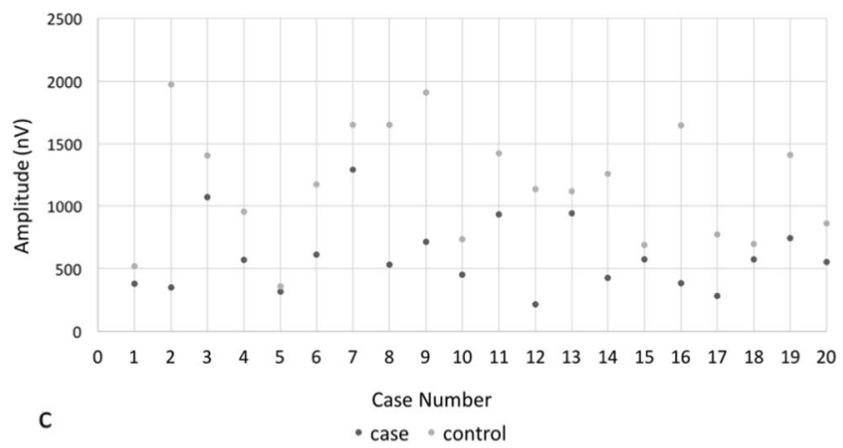

Fig. 2 Box plot representation of P1 wave (a) amplitude and (b) peak time of different rings of mfERG in cases and controls at baseline (The box represents the first quartile, mean and third quartile with standard

function by measuring local ERG responses from the cone-driven retina, which are predominant in the macula, under light adapted conditions. It helps in spatial localisation of the retinal dysfunction [16].

We had previously evaluated the role of mfERG in diagnosing retinal toxicity from siderosis in 6 eyes with normal ERG and no clinical evidence of siderosis [13]. A significant delay was found in group averaged peak time in the affected eyes compared to the fellow eyes at most of the rings $\left(<2^{\circ}, 2-5^{\circ}\right.$, and $>15^{\circ}$ rings $)(p<0.05)$. The average peak time for the overall retinal area also showed significant prolongation $(p=0.010)$.

In this study, mfERG was affected in all eyes with IOFB in terms of both $\mathrm{P} 1$ and $\mathrm{N} 1$ amplitude and peak time at baseline. The mfERG changes were probably due to subclinical siderosis, as the cases did not have any media opacity associated with the trauma (cataract/ vitreous haemorrhage) or macular changes (hole/haemorrhage/ scarring) that could result in similar findings. This study confirms the findings of our previous case series that mfERG has higher sensitivity when compared to ERG in detecting ongoing retinal dysfunction secondary to ocular siderosis. However, it is uncertain that this investigative modality can be satisfactorily used as a diagnostic modality for ocular siderosis

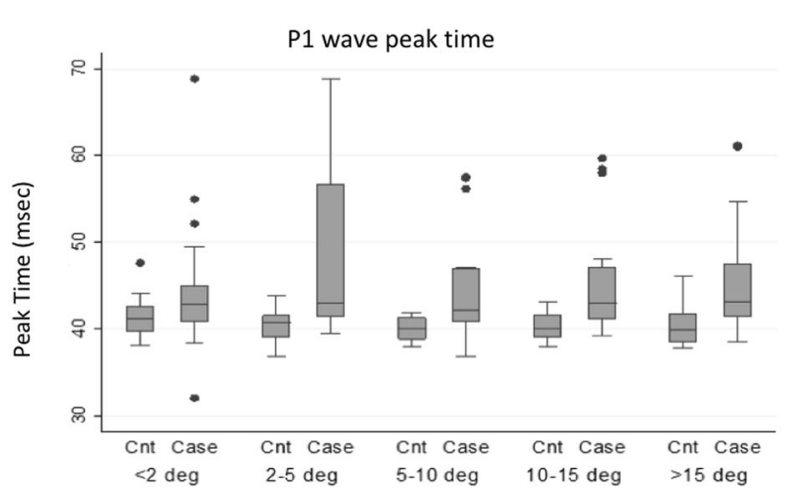

b

Retinal rings of $\mathrm{mfERG}$

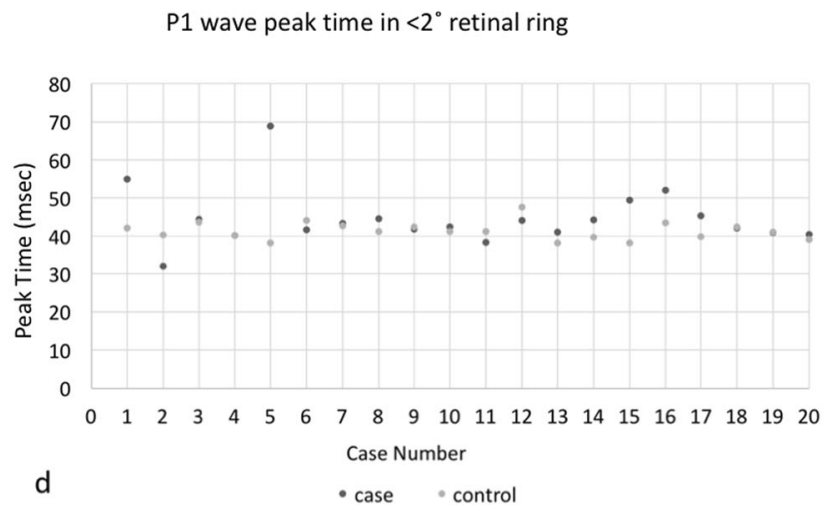

error bar on either side. The dot represents the outliers.), Scatter plot representation of P1 wave (c) amplitude and (d) peak time in the $<2^{\circ}$ retinal ring

considering the high variability in its results. This difference in the outcome of ERG and mfERG may be indicative of differential damage to cones at macula by the ferrous ions contained within the FB. The greater susceptibility of cones compared with rods to the oxidative insult caused by ferrous ions has been established in mice models [19].

Furthermore, this study purports that late presentation following injury may cause greater retinal dysfunction and poor recovery following surgery - cases that presented a month after trauma had lower P1 wave amplitude and much more prolonged peak time as compared to those presenting within a month. This can be explained by the progressive ferrous ion release from the foreign body which gradually increases the oxidative stress in the retina and leads to the electrophysiological changes $[2,20]$. The primary site of dysfunction in ocular siderosis is the RPE. The dysfunction of RPE, in turn, affects the functioning of photoreceptors which are involved in the origin of the N1 wave on mfERG [6]. Changes obtained in P1 wave can be explained by the dysfunction of the bipolar cell layer which largely shapes the P1 wave [6].

The P1 and N1 wave amplitudes were reduced in all the five retinal rings with the inner retinal rings being more affected than the outer retinal rings at baseline. The high 
density of RPE and photoreceptors in the fovea which decreases on moving to the periphery may explain the greater susceptibility of the inner retinal rings in $\mathrm{P} 1$ as well as N1 wave.

The eyes in which silicone oil was used for endotamponade had a much severely affected mfERG at baseline than those in which gas was used. This difference may be explained by the greater severity of retinal damage in these eyes with retinal breaks or localised subretinal fluid noticed intra-operatively for which silicone oil was preferred as the endotamponade agent over gas. Silicone oil replacement of vitreous cavity has been known to have a significant electrical attenuating effect on ERG [21]. Insulating silicone oil disrupts the electrical conduction of retinal currents through the vitreous cavity. This insulating effect of silicone oil may also be the reason for persistently depressed amplitude and delayed peak time of P1 wave on mfERG in oil group on follow up.

Reversal of retinal electrophysiological changes on ERG following surgical removal of FB has been described previously [2, 4]. In the absence of further free ferrous ion release, depending upon the capacity of the retina, macrophages may detoxify and store the remnant free ions. Similarly, in this study mfERG changes reversed partially after surgical removal of FB. The mean amplitude of P1 wave showed significant improvement from baseline on follow-up but did not recover completely till 6 months postoperatively. On the other hand, the amplitude of $\mathrm{N} 1$ and peak time of both $\mathrm{P} 1$ and N1 wave continued to remain deranged on follow-up. Early removal of FB results in better final retinal electrophysiological activity than late presentation and late removal.

The study carries a few limitations. There is no control group with non-iron IOFB to suggest if these changes are only due to siderosis and not due to retinal dysfunction resulting from ocular trauma. Also, a lot of variabilities were noted in the results of mfERG in eyes with IOFB. Hence, further studies are needed in this regard to validate our findings.

From this study, it can be suggested that patients with iron IOFB having a normal ERG may have subclinical electrophysiological changes on mfERG examination. The peak time of P1 wave may serve as a permanent marker for evidence of past retinal dysfunction due to iron IOFB.

\section{Summary}

\section{What was known before}

- Full-field electroretinogram (ERG) is the gold standard investigation to assess retinal dysfunction in eyes with retained iron intraocular foreign body.
- The development of changes in ERG has important prognostic implications and is an indication for surgical removal of foreign body.

\section{What this study adds}

- Multifocal ERG (mfERG) may be abnormal in eyes with iron foreign body even if ERG is normal.

- Defect in mfERG (particularly peak time) may persist even after removal of foreign body.

- Delay of peak time long after foreign body removal can help judge the severity of the injury.

Acknowledgements We sincerely thank Mrs Veena Pandey for her valuable support in statistical analysis. She works at Dr Rajendra Prasad Centre for Ophthalmic Sciences, All India Institute of Medical Sciences, New Delhi, India.

\section{Compliance with ethical standards}

Conflict of interest The authors declare that they have no conflict of interest.

Publisher's note: Springer Nature remains neutral with regard to jurisdictional claims in published maps and institutional affiliations.

\section{References}

1. Sandhu HS, Young LH. Ocular siderosis. Int Ophthalmol Clin. 2013;53:177-84.

2. Henry Kimpton, Duke-Elder S. System of Ophthalmology. 1972;14:525-44.

3. Karpe G. Early diagnosis of siderosis retinae by the use of electroretinography. Doc Ophthalmol. 1948;2:277-96.

4. Kuhn F, Witherspoon CD, Skalka H, Morris R. Improvement of siderotic ERG. Eur J Ophthalmol. 1992;2:44-45.

5. Scholl HP, Zrenner E. Electrophysiology in the investigation of acquired retinal disorders. Surv Ophthalmol. 2000;45:29-47.

6. Hood DC, Frishman LJ, Saszik S, Viswanathan S. Retinal Origins of the primate multifocal ERG: implications for the human response. Invest Ophthalmol Vis Sci. 2002;43:1673-85.

7. Gerth C, Delahunt PB, Alam S, Morse LS, Werner JS. ConeMediated multifocal electroretinogram in age-related macular degeneration: Progression over a long-term follow-up. Arch Ophthalmol. 2006;124:345-52.

8. Bearse MA, Adams AJ, Han Y, et al. A Multifocal electroretinogram model predicting the development of diabetic retinopathy. Prog Retin Eye Res. 2006;25:425-48.

9. Si Y, Kishi S, Aoyagi K. Assessment of macular function by multifocal electroretinogram before and after macular hole surgery. Br J Ophthalmol. 1999;83:420-4.

10. Penrose PJ, Tzekov RT, Sutter EE, et al. Multifocal electroretinography evaluation for early detection of retinal dysfunction in patients taking hydroxychloroquine. Retin Philos Pa. 2003;23: 503-12.

11. Praidou A, Hagan R, Newman W, Chandna A. Early diagnosis of Stargardt disease with multifocal electroretinogram in children. Int Ophthalmol. 2014;34:613-21. 
12. Dolan FM, Parks S, Hammer H, Keating D. The wide field multifocal electroretinogram reveals retinal dysfunction in early retinitis pigmentosa. Br J Ophthalmol. 2002;86:480-1.

13. Gupta S, Midha N, Gogia V, et al. Sensitivity of multifocal electroretinography (mfERG) in detecting siderosis. Can J Ophthalmol. 2015;50:485-90.

14. Kuhn F, Morris R, Witherspoon CD, et al. A standardized classification of ocular trauma. Ophthalmology. 1996;103:240-3.

15. McCulloch DL, Marmor MF, Brigell MG, et al. ISCEV Standard for full-field clinical electroretinography (2015 update). Doc Ophthalmol. 2015;130:1-12.

16. Hood DC, Bach M, Brigell M, Keating D, et al. ISCEV Standard for clinical multifocal electroretinography (2011 edition). Doc Ophthalmol. 2012;124:1-13.
17. Imaizumi M, Matsumoto CS, Yamada K, et al. Electroretinographic assessment of early changes in ocular siderosis. Int $\mathrm{J}$ Ophthalmol. 2000;214:354-9.

18. Lim LT, Shankar V, Blum RA, Hammer HM. Long-standing ironcontaining intraocular foreign body without siderosis. Clin Exp Optom. 2011;94:387-8.

19. Rogers BS, Symons RC, Komeima K, et al. Differential sensitivity of cones to iron-mediated oxidative damage. Invest Ophthalmol Vis Sci. 2007;48:438-45.

20. Knave B. Electroretinography in eyes with retained intraocular metallic foreign bodies. Acta Ophthalmol. 1969;100(suppl):4-63.

21. Michael J, Doslak. A. Theoretical study of the effect of silicone oil on the electroretinogram. Invest Ophthalmol Vis Sci. 1988;29: $1881-4$. 